\title{
Dorsomedial Prefrontal Cortex Mediates Rapid Evaluations Predicting the Outcome of Romantic Interactions
}

\author{
Jeffrey C. Cooper, ${ }^{1,3}$ Simon Dunne, ${ }^{1,3}$ Teresa Furey, ${ }^{1}$ and John P. O’Doherty ${ }^{1,2,3}$ \\ ${ }^{1}$ Trinity College Institute of Neuroscience and ${ }^{2}$ School of Psychology, Trinity College Dublin, Dublin 2, Ireland, and ${ }^{3}$ Department of Computation and \\ Neural Systems, California Institute of Technology, Pasadena, California 91125
}

Humans frequently make real-world decisions based on rapid evaluations of minimal information; for example, should we talk to an attractive stranger at a party? Little is known, however, about how the brain makes rapid evaluations with real and immediate social consequences. To address this question, we scanned participants with functional magnetic resonance imaging (fMRI) while they viewed photos of individuals that they subsequently met at real-life "speed-dating" events. Neural activity in two areas of dorsomedial prefrontal cortex (DMPFC), paracingulate cortex, and rostromedial prefrontal cortex (RMPFC) was predictive of whether each individual would be ultimately pursued for a romantic relationship or rejected. Activity in these areas was attributable to two distinct components of romantic evaluation: either consensus judgments about physical beauty (paracingulate cortex) or individualized preferences based on a partner's perceived personality (RMPFC). These data identify novel computational roles for these regions of the DMPFC in even very rapid social evaluations. Even a first glance, then, can accurately predict romantic desire, but that glance involves a mix of physical and psychological judgments that depend on specific regions of DMPFC.

\section{Introduction}

In a complex social world, humans frequently make rapid evaluations about others on the basis of minimal information, such as facial appearance (Ambady and Rosenthal, 1992; Hassin and Trope, 2000; Willis and Todorov, 2006). Real social behavior, however, requires using these evaluations to make rapid decisions about individuals; for example, should we talk to an attractive stranger at a party who approaches us after making eye contact? These judgments are not trivial; rapid evaluations can have surprisingly large effects on outcomes like wages, judicial decisions, and elections (Zebrowitz and McDonald, 1991; Hamermesh and Biddle, 1994; Todorov et al., 2005).

These evaluations draw in part on perceptions of complex personality traits like trustworthiness and competence, and some insight has been gained into specific neural circuits that support these perceptions, especially in the medial prefrontal cortex (Adolphs et al., 1998; Winston et al., 2002; O'Doherty et al., 2003b; Spezio et al., 2008; Schiller et al., 2009; Rule et al., 2011). The medial prefrontal cortex is thought to support social evaluations along several distinct dimensions, from physical attractiveness to psychological similarity (Amodio and Frith, 2006; Satpute and Lieberman, 2006). Because these studies have fo-

Received May 24, 2012; revised July 28, 2012; accepted Aug. 25, 2012.

Author contributions: J.C.C. and J.P.O. designed research; J.C.C., S.D., and T.F. performed research; J.C.C., S.D., T.F., and J.P.O. analyzed data; J.C.C. and J.P.O. wrote the paper.

This work was supported by an Irish Research Council on Science, Engineering, and Technology fellowship to J.C.C., a Wellcome Trust project grant, and a Gordon and Betty Moore Foundation grant to J.P.O. We gratefully acknowledge technical assistance from Sojo Joseph and research assistance from Jamie Gallagher, Betsy Carroll, and the Science Gallery.

Corresponding author: Jeffrey C. Cooper, Department of Computation and Neural Systems, California Institute of Technology, Pasadena, CA 91125. E-mail: jeff.cooper@gmail.com.

DOI:10.1523/JNEUROSCI.2558-12.2012

Copyright $\odot 2012$ the authors $\quad 0270-6474 / 12 / 3215647-10 \$ 15.00 / 0$ cused on hypothetical interactions or evaluations of strangers, however, little is known about the brain systems involved in making rapid evaluations with real and immediate social consequences, such as deciding to pursue a romantic relationship.

To investigate the systems involved in rapid evaluations for real-world social interactions, we used a novel real-world "speeddating" paradigm (see Fig. 1A) (Kurzban and Weeden, 2005; Finkel and Eastwick, 2008). Participants were scanned with functional magnetic resonance imaging (fMRI) while they viewed photos of individuals that they would subsequently meet at a speed date. The goal of the study was to understand how rapid judgments made from facial appearances influenced subsequent real-world interactions, and to identify the brain systems that mediated these socially relevant judgments.

First, we asked heterosexual participants $[N=151 ; 78$ women (W), 73 men $(\mathrm{M})]$ to rate photos of other opposite-sex participants on three dimensions: a "first-impression" (FI) rating with the scale "How much would you like to date this person?," as well as separate ratings of physical attractiveness (Att) and likability (Like). A subset of participants $(N=39 ; 19 \mathrm{~W}, 20 \mathrm{M})$ were scanned with $\mathrm{fMRI}$ during the FI ratings to measure neural activity during the initial evaluations.

Several days after this presession, participants then attended one or more speed-dating events, at which each participant met $\sim 20$ opposite-sex participants for short conversations and sorted them into two groups: one group, consisting of at least half the partners, who the participant would be interested in seeing again ("pursue"), and the other group, who he or she would not want to see again ("reject"). Those decisions mattered; participants who "matched" (chose to pursue each other) received each other's contact information and could thus initiate further social contact. We hypothesized that distinct evaluations of physical attrac- 
tiveness and psychological compatibility would correlate with subsequent decisions to pursue, and that these evaluations would be mediated by distinct neural systems in the medial prefrontal cortex related to value-based decision making (such as ventromedial prefrontal cortex [VMPFC] and paracingulate cortex) and social evaluation (such as rostromedial prefrontal cortex [RMPFC]).

\section{Materials and Methods}

\section{Participants}

One hundred fifty-one student volunteers ( $>85 \%$ Irish) from Trinity College Dublin participated. Participants were screened to be heterosexual and single. All participants provided informed consent as approved by the Research Ethics Committee of the Trinity College School of Psychology.

Participants were assigned to separate scanning $(N=39 ; 19 \mathrm{~W}, 20 \mathrm{M}$; ages from 19-31 years old, $M=21.44)$ or behavioral-only $(N=112 ; 53$ $\mathrm{M}, 59 \mathrm{~W}$; ages from $18-32$ years old, $M=20.46$ ) pools at signup. Scanning participants were screened for current psychiatric diagnoses, righthandedness, and MRI contraindications (e.g., claustrophobia); they were paid $€ 40$ plus $€ 20$ for each speed-date event attended. Behavioral-only participants were paid $€ 20$ plus $€ 20$ at their event. Behavioral-only participants were included to provide a sufficient number of partners for scanned participants; they attended identical presessions (unscanned) to ensure similar experiences, but their data are not included in results (except where indicated).

\section{Procedures}

Presessions ( $f M R I)$. Participants first attended a signup session where they had a digital photo taken (face and hair only, with a neutral background); participants were allowed to choose their expression and could repeat their photo until they approved it.

Within 6 weeks of signup, participants attended a presession in the lab. At the presession, participants first performed an FI rating task (Fig. $1 \mathrm{~A}$ ). On each trial, a participant's photo was presented above a four-point rating scale (labeled "not at all," "a little," "somewhat," and "very much") that asked "How much would you like to date this person?" Participants had $4 \mathrm{~s}$ to respond. Photos appeared at the same time as the scale, at the onset of the trial. Trials were separated by an intertrial interval displaying a fixation cross (length 1-12 s, randomly drawn from a truncated Poisson distribution, $M=6 \mathrm{~s}$ ).

Scanning participants performed the FI task while being scanned with fMRI, while behavioral-only participants performed the FI task at a computer; the task was otherwise identical.

Each participant's trials contained photos of all their subsequent partners, and other photos of people they did not meet (as control trials); these trials were not distinguished, and participants were told they would meet some but not all of the people they saw. Behavioral-only participants faced 38-44 trials $(M=41.87, \mathrm{SD}=1.29)$; of those, $15-20$ were subsequent partners $(M=18.57, \mathrm{SD}=1.49)$. Scanning participants had a larger set of partners over multiple events (see below), so they faced $62-84$ trials $(M=82.62, \mathrm{SD}=3.43)$; of those, $20-56$ were subsequent partners $(M=50.62, \mathrm{SD}=8.66)$. Presession order was not correlated with subsequent decision $[\beta=0.00$, standard error of estimate $(\mathrm{SEE})=$ $0.05, \mathrm{~ns}]$.

Following the FI task (and after exiting the scanner if needed), participants performed a separate multirating task outside the scanner with the same set of photos in the same order. On each self-paced trial, participants rated that photo on a series of characteristics with nine-point scales, including two ratings of potential romantic desirability: "How physically attractive is this person?" and "How much do you think you would like this person?" (In this task, participants also made one rating of facial happiness and four ratings of personality traits; in preliminary analyses, none of these ratings were correlated with decision after controlling for the three main ratings, and so they are not analyzed further.)

Stimuli for both tasks were presented with Cogent 2000 (Wellcome Trust Centre for Neuroimaging, London).

\section{Speed dating}

Within $1-14 \mathrm{~d}$ of the presession $(M=5.54)$, participants attended their first speed-dating event (Finkel et al., 2007). Each of the six events in- cluded 31-40 participants $(M=36.83)$ with approximately equal numbers of men and women. Events took place midday in a large open classroom.

Each participant received a packet of blank date records and name tag (with first name and ID number) on arrival. Date records included ratings of a partner's personality traits (not analyzed here), a nine-point rating of romantic desirability with the prompt "I was interested in getting to know this partner better," and the pursue versus reject decision: "Would you be interested in seeing this partner again? (Yes or No)."

During each date, participants had an unconstrained conversation with the partner across from them. Each $5 \mathrm{~min}$, all of the men or all of the women (alternating across events) rotated one partner to their right; before beginning the new date, participants filled out a record for the date just completed, including their decision to pursue or reject. The order of interactions was randomized and uncorrelated with the order of face display in the presession $(\beta=0.04, \mathrm{SEE}=0.12$, ns $)$. Participants were instructed to sort their partners into two groups depending on whether they would be interested in that partner as a prospect for a potential follow-up date, with at least $50 \%$ of partners to be placed into the pursue group and the remaining partners into the reject group. (Every participant except one obeyed the instruction to make pursue decisions for half of his or her partners at each event; that participant fell short only by two and so was left in the analyses.) Participants were not aware of this constraint before the events, and so it did not affect their ratings at the presession. Participants also rated each partner's romantic desirability, which correlated strongly with the decision to pursue versus reject (Know ratings; see Results). After all participants had met each oppositesex partner, participants returned their records and were paid in cash.

To ensure enough data for fMRI, scanning participants each attended three speed-date events on three successive days. Six scanning participants $(5 \mathrm{~W}, 1 \mathrm{M})$ missed one event and attended two instead, while one W missed two events and attended one instead. Behavioral-only participants each attended a single event. Male scanning participants attended the first set of three events and met a new group of female behavioralonly partners at each, while female scanning participants attended the second set of three events and met a new group of male behavioral-only partners at each.

Within 1-2 d after the first set of three events and again after the second set, all participants in that set of events were e-mailed a document with photos of all of their partners, their first names and numbers from the events, and each person's decision for each date. For each "match" partner, an e-mail address was also provided (set up for each participant for the study to forward to their personal address).

Scanning parameters. During the FI task, scanning participants were scanned with a Phillips 3 T MRI scanner using the standard head coil, padded to minimize head motion. Functional images covered the whole brain with 39 contiguous 3.55 -mm-thick axial slices with gradient echo $\mathrm{T} 2^{*}$-weighted echoplanar imaging $(\mathrm{TR}=2 \mathrm{~s}, \mathrm{TE}=28 \mathrm{~ms}, 3 \times 3 \mathrm{~mm}$ in-plane voxel size, $80 \times 80$ matrix). The acquisition plane was tilted $\sim 30^{\circ}$ to the anterior-posterior commissure plane to optimize sensitivity in the ventral prefrontal cortex (Deichmann et al., 2003). Each participant's scan consisted of a single functional run whose length varied depending on the number of trials (314-425 images, $M=415.59)$; the first four were discarded to account for magnetic equilibration. Participants also had a high-resolution structural image taken before the task began (3D acquisition; T1-weighted spoiled gradient-recalled echo sequence; $0.9 \times 0.9 \times 0.9 \mathrm{~mm}$ voxel size; $256 \times 256 \times 180$ matrix).

Statistical analysis. Data were analyzed with MATLAB (The Mathworks) and SPM8 (Wellcome Department of Imaging Neuroscience; London). All tests were two-tailed. Hierarchical linear models were fit using the nlmefit command using maximum likelihood and a logistic link function for decisions, nesting ratings or decisions within participants. Hierarchical models included random intercepts; preliminary analysis indicated no other random effects were significant and so they were not included. All predictors for all models were $Z$-scored over the whole group before entering the model. Classification accuracy was tested with leave-one-participant-out cross-validation.

Spatial preprocessing. Functional images were preprocessed with standard parameters, including slice timing correction (to the center slice), 
realignment (to each participant's first image), coregistration of the highresolution structural image, segmentation of the structural image into tissue types (using the "New Segment" routine with the default templates), spatial normalization of the functional images (into MNI space, using parameters estimated from the segmented structural image and SPM8 default normalization parameters), and spatial smoothing (with a $4 \mathrm{~mm}$ full-width at half-maximum Gaussian kernel).

Neuroimaging models. All models were estimated using restricted maximum likelihood and an AR(1) model for temporal autocorrelation, as standard in SPM8. A highpass filter (cutoff $128 \mathrm{~s}$ ) removed lowfrequency noise. All models contained six predictors of no interest that encoded residual head motion as well as a constant term. Trials were specified as delta function regressors of $0 \mathrm{~s}$ duration with onset at the beginning of the trial. All models also included a separate predictor for control faces (i.e., participants who the scanned participant did not meet); this predictor was not analyzed.

Four neuroimaging models were estimated for the main results. The first, basic model (Figs. 2A, 3; Table 2), included two predictors of interest: partners who were subsequently pursued and partners who were subsequently rejected. The second model controlled addressed this main contrast but controlled for reaction time (Table 2). It included a single predictor for all partners with two parametric modulators: one for reaction time in responding to the FI measure, followed by a contrast-coded modulator comparing subsequently pursued versus rejected partners. The third model addressed which regions correlated with subjective desirability ratings (Figs. $2 B, C, 3$; Table 2). It included a single predictor for all partners with two parametric modulators: one for the Att rating (subjective physical attractiveness of that partner), followed by one for the Like rating (subjective likeability of that partner). The fourth model, adjusted for partner and relationship effects (Fig. 4; Table 4), included a single predictor for all partners with two parametric modulators: one for the decision consensus judgment (the average decision to pursue or reject for each partner over all participants, with pursue $=1$ and reject $=$ 0 ), followed by one for the decision individual preference (the participant's decision to pursue or reject for that partner minus the consensus judgment for that partner). As is standard in SPM8, all parametric modulators were orthogonalized with respect to all modulators that preceded them in the model, and hence were controlled for the effects of all preceding modulators.

For additional tables and results, an additional three models were estimated. For activation correlated with FI ratings (see Results), the model included a single predictor for all partners, with one parametric modulator for the FI rating. The other two models were used to investigate activation correlated with Know ratings (see Results). One model (Fig. 5A; Table 2) included a single predictor for all partners, with a single parametric modulator for Know ratings. The last model (Fig. $5 B, C$; Table 4) included a single predictor for all partners, with parametric modulators for the Know consensus judgment (average Know rating for each partner over all participants) and the Know individual preference (the participant's Know rating minus the consensus judgment).

Participants' parameter-estimate images were carried forward to random effects analyses and tested with one-sample $t$ tests across the group. Activations were thresholded voxelwise at $p<0.001$ and with an extent threshold based on Gaussian random fields set to control the wholebrain family-wise error rate (FWE) at $p<0.05$ (Worsley et al., 1996); this cluster threshold varied between 21 and 25 voxels $\left(671-799 \mathrm{~mm}^{3}\right)$. Because this cluster threshold was large enough to potentially screen out some small subcortical regions, we conducted preliminary analyses with a more liberal cluster threshold (10 voxels). At this exploratory threshold, no clusters emerged in both the decision-based and Know-rating based version of any key contrast, so we do not report any results at this threshold.

Region of interest analyses. For contrasts with multiple activated clusters (e.g., for partners who were later pursued instead of rejected), we compared how these clusters were independently correlated with subsequent decisions using hierarchical linear models with each cluster's activation time course as a separate predictor. Each time course was extracted from a $4 \mathrm{~mm}$ radius sphere centered on the cluster's peak, converted to percentage signal change from the mean, linearly de- trended, and highpass filtered (128 s window); three time points were entered in as separate predictors for each trial for each cluster (at 4,6, and $8 \mathrm{~s}$ following trial onset, to account for the hemodynamic delay). Models were then fit identically to behavioral hierarchical models (logistic regression, random intercepts and $Z$-scoring over the group).

To estimate overall effect sizes between conditions from functional regions of interest (Figs. 3, 4B, 5C), we used leave-one-out extraction to provide an independent criterion for voxel selection (Kriegeskorte et al., 2009): for each participant, $\beta$ weights were extracted from significant voxels for that cluster or region of interest in a group model excluding that participant using rfxplot (Gläscher, 2009).

\section{Results}

\section{Behavioral}

\section{Pursuit rates}

Scanned participants made decisions to pursue $59.4 \%$ of their partners on average ( $\mathrm{SEM}=1.5 \%)$, and pursuit rates ranged from 47.2-96.2\%. Pursuit rates for women and men did not differ significantly $\left(t_{(37)}=0.20\right.$, ns; women's $M=59.7 \%$, SEM $=2.7 \%$; men's $M=59.1 \%$, SEM $=1.5 \%)$. Behavioralonly participants' pursuit rates did not significantly vary from scanned participants $\left(M=57.7 \%, \mathrm{SEM}=0.9 \% ; t_{(149)}=0.95\right.$, ns). (Behavioral participants are not included in further results; their behavioral results were extremely similar to scanned participants' results.)

\section{Presession ratings}

We first examined the extent to which the FI measure was related to the subsequent decision to pursue or reject a potential partner at the speed-date events (Fig. $1 B$; Table 1). The FI measure was highly positively correlated with subsequent decisions in a hierarchical linear model $(t=9.44, p<0.001$; cross-validated model accuracy $=61.6 \%$, SEM $=1.1 \%$ ), suggesting that participants were able to judge well above chance which partners they would find desirable from viewing photos for only a few seconds several days earlier.

Next, we examined the extent to which decisions were driven by subjective judgments of physical attractiveness compared with likability. We found that decisions were significantly correlated with both ratings of Att and Like when each were included alone in a hierarchical linear model (Fig. $1 B$; Att: $t=10.06, p<0.001$, model accuracy $=61.8 \%, \mathrm{SEM}=1.3 \%$; Like: $t=9.50, p<0.001$, model accuracy $=61.9 \%$, SEM $=1.4 \%$ ).

Because Att and Like ratings were also highly correlated with each other (mean within-participant $r=0.68$, SEM $=0.03$ ), we examined the unique contribution of each measure by including both in the model (Table 1). Although both ratings were significant, Att ratings accounted for a notably larger share of variance in decisions (Att: $\beta=0.39, \mathrm{SEE}=0.08, t=5.09, p<0.001$; Like: $\beta=0.27, \operatorname{SEE}=0.07, t=3.64, p<0.001$; model accuracy $=$ $62.1 \%$, SEM $=1.3 \%)$.

Att and Like ratings were also highly correlated with the FI judgment itself (Att-FI: mean within-participant $r=0.66$, $\mathrm{SEM}=0.02$; Like-FI: mean within-participant $r=0.53$, SEM $=$ 0.02 ), and partial correlations revealed that Att ratings accounted for the majority of the variance underpinning FI ratings (mean within-participant Att-FI partial correlation controlling for Like $=0.46$, SEM $=0.02$; mean within-participant Like-FI partial correlation controlling for Att $=0.15, \mathrm{SEM}=0.02$ ).

In all models, neither the main effect of participant gender nor any interaction with gender was significant, consistent with earlier studies suggesting men and women rely on similar judgments to make real-world speed-dating decisions (Eastwick and Finkel, 2008). These analyses therefore indicated that a substantial por- 
A
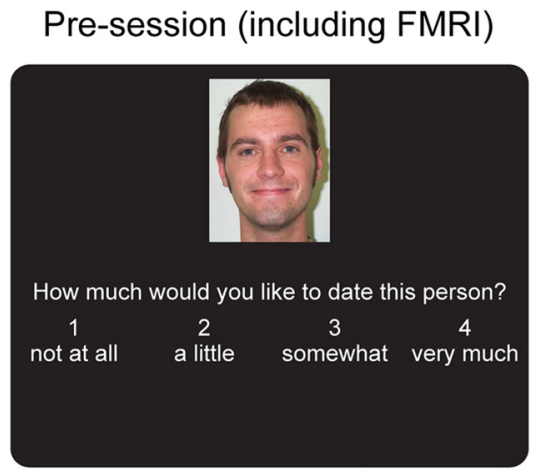

First-impression task (scanned)

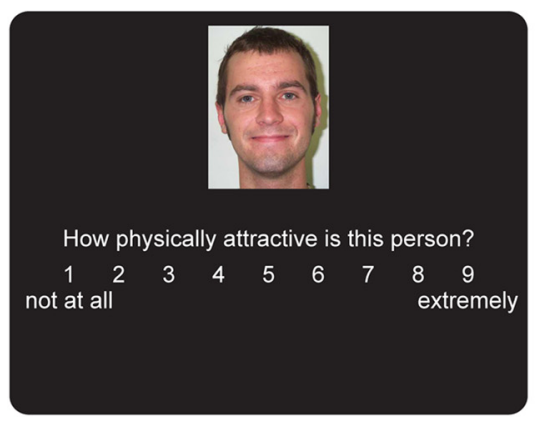

Multi-rating task (not scanned)

B
0
$\frac{0}{0}$
$\frac{0}{0}$
$\frac{0}{0}$
0
0
$\frac{0}{0}$
$\frac{10}{5}$
$\frac{2}{4}$
0
0
0
$\frac{\pi}{0}$
0
0
0
0

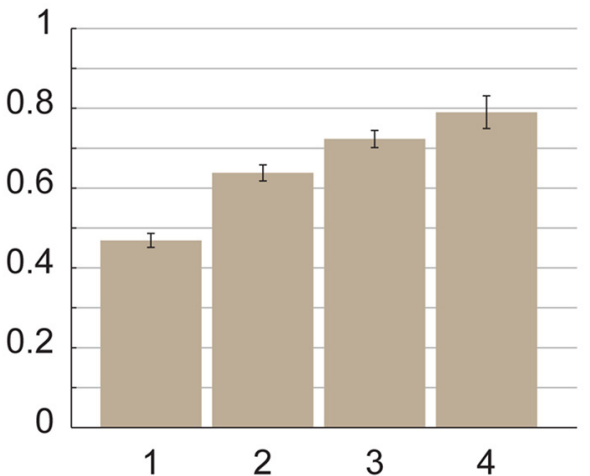

First-impression (FI)

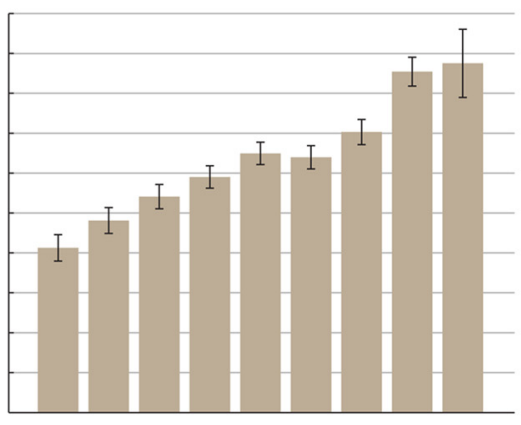

$\begin{array}{lllllllll}1 & 2 & 3 & 4 & 5 & 6 & 7 & 8 & 9\end{array}$

Att

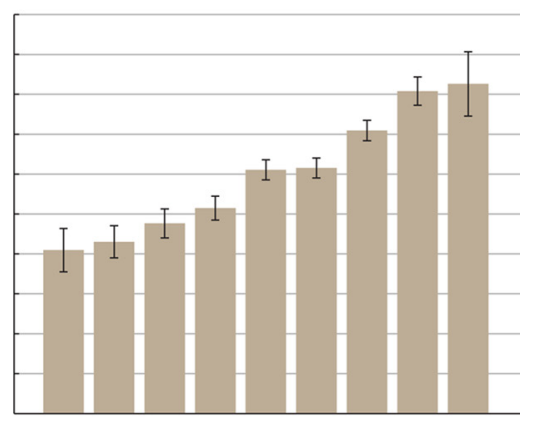

$\begin{array}{lllllllll}1 & 2 & 3 & 4 & 5 & 6 & 7 & 8 & 9\end{array}$

Like

Figure 1. Design and behavioral results. $A$, Experimental design. At a presession, participants ( $N=39 \mathrm{for}$ fMRI scanning) rated photos of potential romantic partners (other participants) in two separate tasks. First, during fMRI scanning, each FI trial asked for one Fl rating; participants had $4 \mathrm{~s}$ to respond (trials separated by $1-12$ s interval). Next, outside the scanner, each multirating trial asked for several self-paced ratings, including two desirability ratings: physical attractiveness (Att, shown) and likability (Like). Presessions were followed (1-14 $\mathrm{d}$ later) by speed-dating events ( $\sim 20$ of each gender at each) where scanned participants met other participants ( $N=112$, behavioral-only) for 5 min conversations and made "pursue" or "reject" decisions about whether they wanted to see each partner again. Mutual pursue decisions received each other's contact information following the events. Diagram shows event layout (each "date" at separate table, with one gender rotating after each date). Sample photo courtesy of (enter for Vital Longevity Face Database. $\boldsymbol{B}$, Decision rates by desirability ratings. Bars are percentage of pursue decisions given to partners receiving that rating for each scale (scanned participants only, $N=39$ ). Error bars indicate $S E s$ across all observations.

Table 1. Relationships between decisions and behavioral ratings

\begin{tabular}{lllll}
\hline & Fl only & Att only & Like only & Att + Like \\
\hline $\mathrm{FI}$ & $0.53^{* * *}(0.06)$ & - & - & - \\
Att & - & $0.59^{* * *}(0.06)$ & - & $0.39^{* * *}(0.08)$ \\
Like & - & - & $0.52^{* * *}(0.05)$ & $0.27^{* * *}(0.07)$ \\
BIC & 2622.36 & 2616.02 & 2628.36 & 2605.06 \\
Accuracy (\%) & $61.6(1.1)$ & $61.8(1.3)$ & $61.9(1.4)$ & $62.2(1.3)$ \\
\hline
\end{tabular}

$\beta$ weights for hierarchical multiple regression of three desirability ratings on speed-dating decisions within scanning participants $(N=39)$. Different columns indicate separate models. Parenthetical values are SEEs. Intercepts not shown for clarity. BIC, Bayesian information criterion; Accuracy, leave-one-out cross-validated model accuracy as percentage correct (parenthetical value is SEM accuracy). ${ }^{* * *} p<0.001$. tion of pursue versus reject decisions could be predicted by the initial subjective impressions obtained from merely viewing a potential partner's picture days earlier. Further, a participant's subjective physical attractiveness rating for a prospective date was by far the most significant predictor of subsequent pursuit (Eastwick and Finkel, 2008).

\section{fMRI}

Activation for pursued versus rejected partners In the fMRI data, we first tested for brain regions showing differential activity for viewing pictures of partners who were subsequently pursued versus rejected at the speed-date events. We 
A
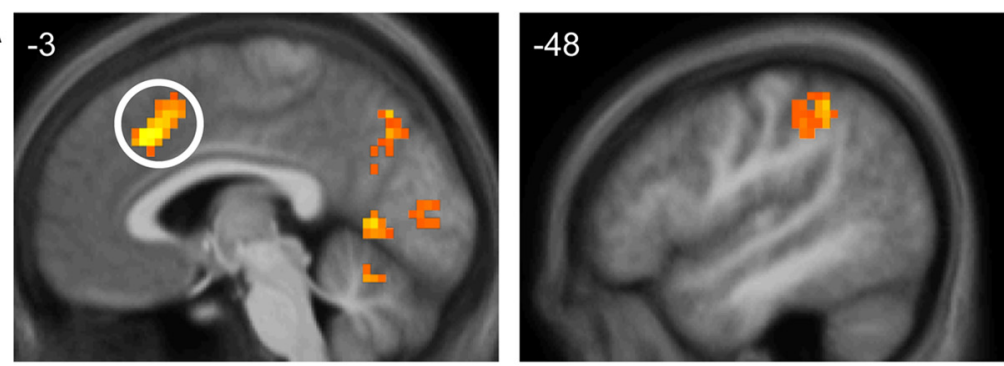

B -3
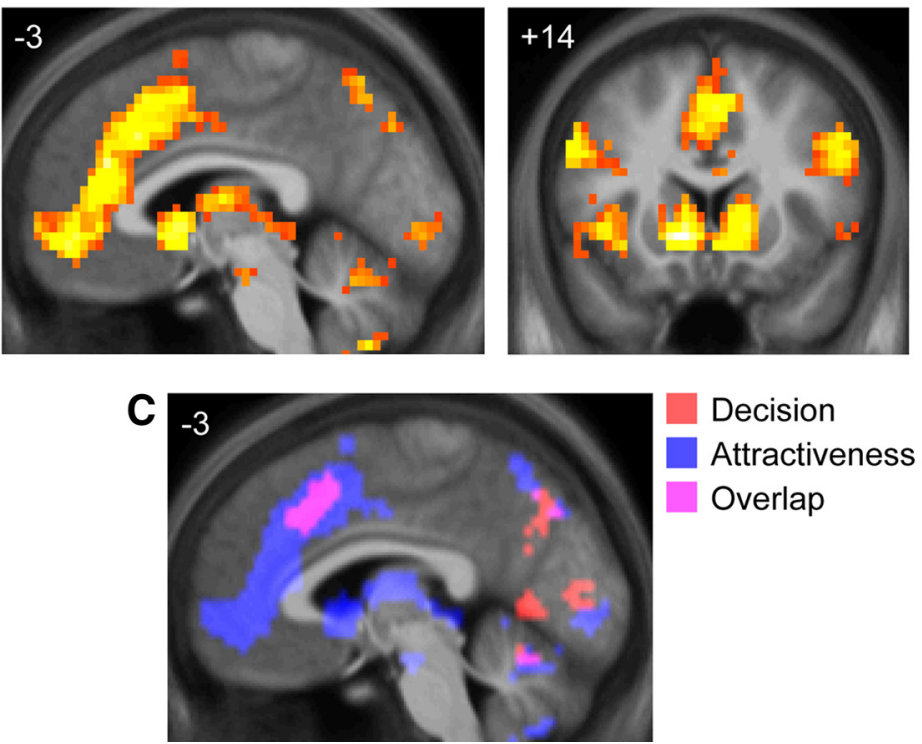

Figure 2. Neural predictors of subsequent decision compared with areas mediating judgments of physical attractiveness. $A$, Brain regions showing greater responses at the time of first viewing for faces of individuals that are subsequently (after speed dating) selected as a potential romantic partner ("pursued"), compared with those who were not ("rejected"). Paracingulate cortex (circled) is the only activated region that significantly independently correlates with subsequent decision in a multiple regression including all activated regions. $\boldsymbol{B}$, Brain regions positively correlating with subjective ratings of physical attractiveness for each partner (Att). $C$, $O$ verlap between brain regions related to decision and those related to attractiveness, showing substantial overlap between these variables in the paracingulate cortex. All images thresholded at $p<0.001$ voxelwise with extent threshold set to control whole-brain FWE at $p<0.05$. Color bars indicate $t$ statistic. Coordinates in ICBM/MNI space.

found greater activation for partners who were ultimately pursued than for individuals who were ultimately rejected in four clusters, including a region of paracingulate cortex (Fig. 2A; Table 2). (Nearly identical regions were correlated with the continuous rating of romantic desirability at the event; Fig. 5A; Table 2.) In a multiple regression of activation time courses from all four clusters predicting subsequent decision, only the paracingulate was significantly correlated with decision $(\beta=0.14, \mathrm{SEE}=0.06, p=0.02)$, indicating this region was the most important independent contributor to later decisions. Furthermore, to control for the prosaic possibility that the activation in paracingulate cortex reflected the difficulty or conflict involved in choosing a rating in the scanner as opposed to an evaluation related to romantic desirability, we analyzed a separate model that included reaction time as a regressor of no interest. Even after adjusting for the effects of reaction time, the paracingulate cortex was still significantly activated for subsequently pursued versus rejected partners (Table 2).

\section{Activation for attractiveness and likability}

Next, we investigated whether these decision-related activations could be accounted for by neural responses elicited by subjective judgments of physical attractiveness, as this was the primary behavioral predictor of subsequent dating decisions. Att ratings were significantly positively correlated with activity in a number of reward-
6

6

4

related brain areas, consistent with previous studies (Aharon et al., 2001; O'Doherty et al., 2003b; Bray and O'Doherty, 2007), including the VMPFC and ventral striatum, as well the paracingulate gyrus and sulcus (Fig. 2B,C; Table 2). (Perhaps unsurprisingly, given the high correlation between attractiveness and first impressions, an extremely similar and overlapping network of regions was activated in a separate model including only the FI rating made during the scan.) After accounting for activity correlating with attractiveness, however, no regions were significantly correlated with likability ratings.

Both the VMPFC and the paracingulate cortex were thus significantly activated by subjective physical attractiveness. In a multiple regression predicting Att ratings using activation time courses from these two regions together, both regions were significantly correlated, indicating that each region correlated with independent variation related to physical attractiveness (Paracingulate $\beta=0.17$, SEE $=0.05, p<$ 0.001 ; VMPFC $\beta=0.17$, SEE $=0.05, p<$ $0.001)$. In contrast, in a multiple regression predicting subsequent decisions with these two regions together, only the paracingulate cortex was significantly correlated, indicating it is a better independent predictor of decision than VMPFC (Paracingulate $\beta=0.17$, SEE $=0.06, p=0.004$; VMPFC $\beta=-0.06$, SEE $=0.06$, ns). Examining activation for these regions for photos of different physical attractiveness (Fig. 3), VMPFC activation increased for partners with very high Att ratings but did not strongly distinguish between those with lower Att ratings; in contrast, paracingulate activation was correlated with differences between medium and low attractiveness, an important difference for speed-dating decisions.

\section{Activation for consensus and individual preferences}

We next investigated which neural systems involved in the speeddate decision reflected effects related to group consensus about a partner's desirability, as opposed to those related to idiosyncratic judgments unique to a particular participant. For this we divided the dating decision and subjective rating variables into two frequently studied components (Kenny and Albright, 1987; Kenny, 1994; Eastwick et al., 2007; Wood and Brumbagh, 2009): a consensus judgment effect corresponding to the average decision or rating made about a partner across all participants, and an individual preference effect measured by the difference between the actual decision or rating made for an individual and the consensus judgment for that individual. Consensus judgment and individual preferences could then be analyzed separately to investigate how each was related to separate neural signals.

In the current study, consensus decisions - the average decision made about each partner-ranged widely (range $=0.05$ 1.0 , or receiving $5-100 \%$ Yes decisions), but they were not dominant; consensus decisions accounted for only $26.1 \%$ of the total decision variance. This is consistent with earlier studies sug- 
Table 2. Activations correlated with subsequent decisions/ratings

\begin{tabular}{|c|c|c|c|c|c|}
\hline Region & $\begin{array}{l}\text { Peak } \\
\text { Z-score }\end{array}$ & $x$ & $y$ & $z$ & $\begin{array}{l}\text { Cluster size } \\
\text { (vox) }\end{array}$ \\
\hline \multicolumn{6}{|l|}{ Decision (yes > no) } \\
\hline Paracingulate cortex & 5.40 & 6 & 20 & 42 & 102 \\
\hline Ventral visual cortex & 4.43 & 6 & -61 & 0 & 74 \\
\hline Medial precuneus & 4.19 & -6 & -70 & 39 & 137 \\
\hline Lateral parietal cortex & 3.99 & -48 & -43 & 49 & 77 \\
\hline $\begin{array}{l}\text { Decision ( } \mathrm{N}_{0}>\text { Yes) } \\
\quad \text { No regions active at this threshold }\end{array}$ & & & & & \\
\hline \multicolumn{6}{|c|}{ Decision controlled for reaction time (Yes $>$ No) } \\
\hline Ventral visual cortex & 4.39 & 0 & -61 & 3 & 96 \\
\hline Paracingulate cortex & 4.37 & 3 & 20 & 42 & 34 \\
\hline Medial precuneus & 3.92 & -9 & -67 & 35 & 136 \\
\hline \multicolumn{6}{|c|}{$\begin{array}{l}\text { Decision controlled for reaction time (No > Yes) } \\
\text { No regions active at this threshold }\end{array}$} \\
\hline \multicolumn{6}{|c|}{ Attractiveness (positive) } \\
\hline Striatum (dorsal and ventral) & 5.97 & -9 & 11 & -4 & 543 \\
\hline Anterior insula & 5.66 & -30 & 29 & 0 & 179 \\
\hline DMPFC & 5.41 & 3 & 17 & 46 & 1024 \\
\hline VMPFC & 5.37 & 3 & 47 & -4 & $*$ \\
\hline Dorsolateral PFC & 5.35 & -30 & -4 & 57 & 544 \\
\hline Medial parietal cortex & 5.23 & -24 & -64 & 35 & 1017 \\
\hline Cerebellum (lateral) & 5.15 & -33 & -61 & -32 & 818 \\
\hline Anterior insula & 4.83 & 36 & 29 & -7 & 165 \\
\hline Medial temporal cortex & 4.59 & -24 & -22 & -15 & 29 \\
\hline Dorsolateral PFC & 4.31 & 51 & 14 & 28 & 137 \\
\hline Dorsolateral PFC & 4.30 & 30 & -4 & 53 & 50 \\
\hline Ventral thalamus & 4.28 & 18 & -25 & -11 & 50 \\
\hline Lateral parietal cortex & 4.23 & 42 & -37 & 46 & 129 \\
\hline Cerebellum (lateral) & 4.14 & -27 & -40 & -36 & 28 \\
\hline Ventrolateral PFC & 3.85 & 42 & 50 & 17 & 30 \\
\hline \multicolumn{6}{|l|}{ Attractiveness (negative) } \\
\hline \multicolumn{6}{|l|}{ Know (positive) } \\
\hline Dorsolateral PFC & 4.49 & -51 & 17 & 35 & 53 \\
\hline Paracingulate cortex & 4.20 & -6 & 17 & 46 & 50 \\
\hline Ventral visual cortex & 4.01 & 0 & -61 & 0 & 25 \\
\hline Medial precuneus/posterior cingulate & 3.94 & 3 & -64 & 25 & 88 \\
\hline Cerebellum (medial) & 3.65 & 6 & -67 & -18 & 25 \\
\hline & \\
\hline
\end{tabular}

DMPFC, Dorsomedial prefrontal cortex; PFC, prefrontal cortex; VMPFC, ventromedial prefrontal cortex; Know, know ratings. Activations in table were thresholded voxelwise at $p<0.001$ and with a cluster size set to control for multiple comparisons over whole brain at $p<0.05\left(23-25\right.$ voxels or $\left.735-799 \mathrm{~mm}^{3}\right)$. $t$ statistics were converted to $Z$-scores for reporting. Coordinates are reported in MNI space, as in SPM8. Voxel size was $3 \times 3 \times 3.2 \mathrm{~mm}$. *Subpeak in above cluster.

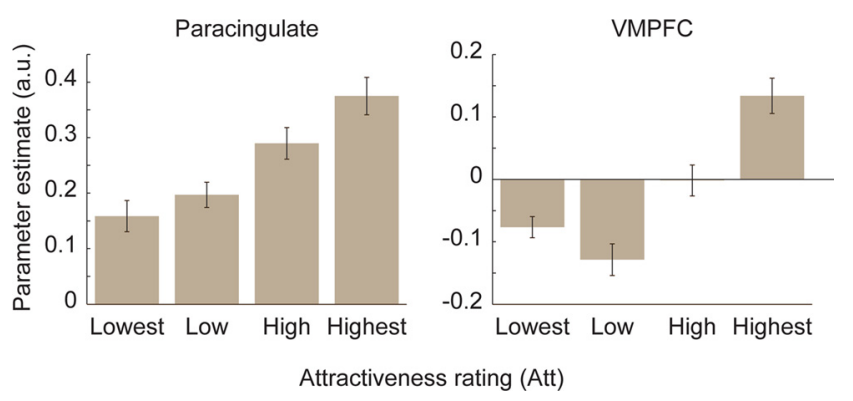

Figure 3. Medial prefrontal activation and Att ratings. Bars indicate average $\beta$ weights for response to partner photos, split by Att rating binned into quartiles within-participants (calculated with leave-one-out extraction; see Materials and Methods). Paracingulate $=$ peak for subsequent decision, $x / y / z=6 / 20 / 42 ;$ VMPFC $=$ peak of correlation with Att in VMPFC, $x / y /$ $z=3 / 47 /-4$. All regions of interest were $4 \mathrm{~mm}$ spheres centered on peak coordinates. Error bars indicate SEM across participants. Significant differences not tested; plots shown to illustrate response pattern only.
Table 3. Relationship between decisions and consensus/individual preference effects

\begin{tabular}{lc}
\hline & Consensus + individual preference \\
\hline Att consensus & $0.48^{* * *}(0.11)$ \\
Like consensus & $0.18(0.11)$ \\
Att individual preference & $0.09(0.07)$ \\
Like individual preference & $0.17^{* *}(0.07)$ \\
BIC & 2549.9 \\
Accuracy (\%) & $63.6(1.1)$ \\
\hline
\end{tabular}

$\beta$ weights for hierarchical multiple regression of consensus and individual preference effects for Att and Like ratings on speed-dating decisions within scanning participants $(N=39)$. Parenthetical values are SEEs. Intercept not shown for clarity. Consensus, Average rating for partner over all participants; individual preference, participant's rating for partner- consensus effect for partner; BIC, Bayesian information criterion; Accuracy, leave-one-out crossvalidated model accuracy (parenthetical value is SEM accuracy). ${ }^{* * *} p<0.001 ; * * 0.01$

gesting that interpersonal liking is dominated not by consensus but by individual preferences (Kenny, 1994; Kenny et al., 2006).

Importantly, including consensus judgments and individual preferences together improved the predictive model for subsequent decisions (Table 3). A hierarchical linear model that included separate predictors for consensus and individual preference effects for Att and Like, compared with the earlier model with undivided Att and Like ratings alone, had better accuracy and model fit (cross-validated accuracy: 63.6\%, SEM = 1.1\%; Bayesian information criterion: 2549.9 vs 2605.06; loglikelihood test: $\left.\chi^{2}(2)=31.49, p<0.001\right)$. In this combined model, the consensus effect of Att was significant while the consensus effect of Like was not; in contrast, only the individual preference effect of Like, and not Att, was significant. This pattern indicates that Att and Like ratings were differentially connected to consensus versus individual preferences about decisions. A partner's average desirability in speed-dating was most related to consensus about her physical attractiveness, and not to consensus about how likeable she seemed. In contrast, a participant's idiosyncratic speed-dating preference for a partner was most related to an individual judgment of how likeable she seemed, and not to an individual judgment of her attractiveness.

Next, brain regions could also be separated by whether they correlated better with consensus or individual preferences; this analysis distinguishes whether a participant's brain region responded to partners who were on average desirable across participants (consensus effects) or who were especially desirable to that specific participant (individual preference effects). In a model including both effects, several regions identified in the original analysis specifically correlated with consensus judgments (Fig. $4 A$; Table 4 ), including the anterior cingulate cortex, medial prefrontal cortex, and cerebellum.

In contrast, individual preferences recruited a single region of dorsomedial prefrontal cortex (DMPFC; Fig. 4B; Table 4), the RMPFC. When the RMPFC was included in a multiple regression for subsequent decisions with the paracingulate cortex, both regions were significantly independently correlated with decisions (paracingulate $\beta=0.16, \mathrm{SEE}=0.06, p=0.005$; $\mathrm{RMPFC} \beta=$ $0.12, \mathrm{SEE}=0.05, p=0.03)$. This pattern suggests that, unlike the VMPFC, RMPFC activation encoded an independent signal that helped predict subsequent decisions, and that this signal was related to a participant's idiosyncratic preferences for particular partners.

\section{Know ratings}

Because participants were asked to pursue at least half of their partners, a pursuit rate above the average for students in similar published studies ( 40\%) (Finkel and Eastwick, 2009), one potential concern is that some partners were chosen to pursue sim- 

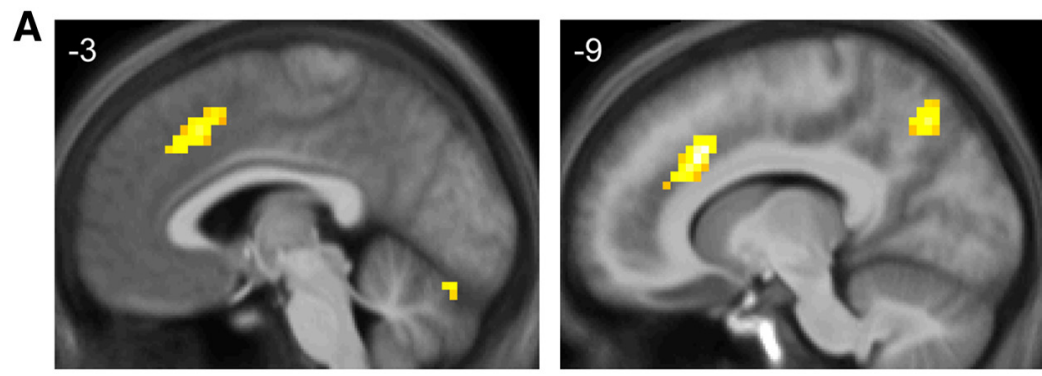

B
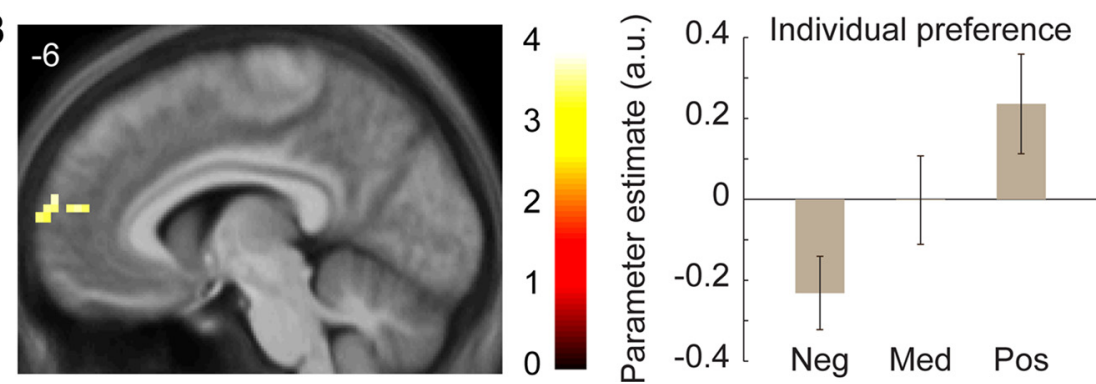

Figure 4. Distinct regions of medial prefrontal cortex mediate effects of consensus judgments and individual preferences. $\boldsymbol{A}$, Region of paracingulate cortex significantly correlated with consensus judgments for decisions (i.e., partners who were more frequently pursued). $\boldsymbol{B}$, A distinct region of RMPFC was correlated with individual preferences for decisions (i.e., individual decisions controlling for consensus judgments). Right, Indicates average $\beta$ weights from significant RMPFC cluster (calculated with leave-one-out-extraction) by size of individual preference effect. Neg, bottom third; Med, middle third; Pos, top third (withinparticipant). Error bars indicate SEM across participants. All images thresholded at $p<0.001$ voxelwise with extent threshold set to control whole-brain FWE at $p<0.05$. Color bars indicate $t$ statistic. Coordinates in ICBM/MNI space.

Table 4. Activations correlated with consensus judgments and individual preferences

\begin{tabular}{|c|c|c|c|c|c|}
\hline Region & Peak Z-score & $x$ & $y$ & Z & $\begin{array}{l}\text { Cluster size } \\
\text { (vox) }\end{array}$ \\
\hline \multicolumn{6}{|c|}{ Decision consensus (positive) } \\
\hline Paracingulate cortex & 5.05 & 3 & 20 & 39 & 132 \\
\hline Medial precuneus & 4.33 & 12 & -67 & 46 & 37 \\
\hline Ventrolateral PFC & 4.18 & -33 & 50 & 10 & 34 \\
\hline Cerebellum (medial) & 3.89 & 3 & -76 & -39 & 52 \\
\hline Medial precuneus & 3.83 & -9 & -64 & 53 & 29 \\
\hline \multicolumn{6}{|c|}{ Decision individual preference (positive) } \\
\hline RMPFC & 3.81 & 3 & 56 & 17 & 36 \\
\hline \multicolumn{6}{|c|}{ Decision consensus (negative) } \\
\hline \multicolumn{6}{|c|}{ Decision individual preference (negative) } \\
\hline \multicolumn{6}{|c|}{ Know consensus (positive) } \\
\hline Paracingulate cortex & 4.44 & 3 & 20 & 42 & 60 \\
\hline Cerebellum (medial) & 3.91 & -6 & -76 & -18 & 30 \\
\hline \multicolumn{6}{|c|}{ Know individual preferences (positive) } \\
\hline RMPFC & 4.03 & 6 & 53 & 10 & 51 \\
\hline Posterior cingulate & 3.46 & -3 & -49 & 28 & 37 \\
\hline \multicolumn{6}{|c|}{ Know consensus (negative) } \\
\hline \multicolumn{6}{|c|}{ No regions active at this threshold } \\
\hline \multicolumn{6}{|c|}{ Know individual preferences (negative) } \\
\hline No regions active at th & & & & & \\
\hline
\end{tabular}

PFC, Prefrontal cortex; RMPFC, rostromedial prefrontal cortex. Know = Know ratings. Activations in table were thresholded voxelwise at $p<0.001$ and with a cluster size set to control for multiple comparisons over whole brain at $p<0.05\left(21-23\right.$ voxels or $\left.671-735 \mathrm{~mm}^{3}\right)$. t statistics were converted to $Z$-scores for reporting. Coordinates are reported in MNI space, as in SPM8. Voxel size was $3 \times 3 \times 3.2 \mathrm{~mm}$.

ply to follow instructions, potentially biasing the analysis of pursued versus reject partners. To address this concern, we also analyzed the relationship between neural activity and the continuous nine-point scale of romantic desirability made at the events ("I was interested in getting to know this partner better"; Know).

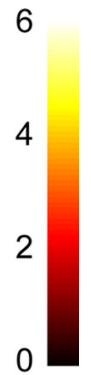

Know ratings were highly correlated with pursue decisions; a hierarchical logistic regression using only this rating correctly classified $84.86 \%$ of decisions. This rating could thus be treated as an unconstrained, continuous version of the pursue versus reject decision. Know ratings were also similarly correlated with Att and Like ratings. As with decisions, both were significantly independently correlated with Know ratings in a hierarchical multiple regression, with Att having a larger effect (Att: $\beta=0.29, \mathrm{SEE}=0.05, t=5.34, p<$ 0.001 ; Like: $\beta=0.21$, $\operatorname{SEE}=0.05, t=$ $4.06, p<0.001)$. The results again indicate that both physical attractiveness and psychological judgments of likability were relevant to real-world judgments of desirability.

Given these ratings' high correlation with decisions, brain activation during viewing of partner photos was unsurprisingly correlated with Know ratings. Activation was positively correlated with Know ratings in a network highly overlapping with the network correlated with subsequent decision (Fig. 5A; Table 2). Similarly, when Know ratings were separately analyzed as consensus judgments and individual preferences, the networks activated in each analysis were highly overlapping with the same networks activated by decision consensus and individual preferences (Fig. 5B,C; Table 4). As the Know ratings were a continuous and unconstrained measure of romantic desirability, these data suggest that the links between desirability ratings and brain activation to real-world speed-dating decisions were unlikely to be significantly driven by the constraint to pursue at least half of one's partners.

\section{Discussion}

To behave adaptively in complex social environments, humans must frequently evaluate others based on little more than a rapid glance at a face or a short conversation. Despite their speed, these rapid evaluations form the basis for real-world social decisions that can have lasting consequences, such as whether to pursue or reject a potential romantic partner. The current study investigated the neural basis of these evaluations by scanning participants with fMRI while they made rapid evaluations of potential romantic partners from their photos several days before meeting them at a real-world "speed-dating" event and deciding whether to pursue or reject each partner.

We found that two distinct regions of DMPFC, the paracingulate cortex and the RMPFC, were significantly predictive of whether a romantic partner would be pursued or rejected. These regions were correlated with separate patterns of behavioral ratings about the partners: paracingulate cortex was best associated with average participant ratings of physical attractiveness and average partner desirability, while the RMPFC was best associated with individual participants' idiosyncratic preferences for specific partners.

These results are the first to link specific regions of DMPFC to consequential real-world social decisions, and together, they shed light on two distinct components of these decisions. One component is a property of the partner himself or herself, and was most 
related to consensus judgments about physical attractiveness. Partners with high attractiveness by consensus received more pursue decisions. These partners also elicited activation in several brain regions associated with reward-related decision making, in particular the paracingulate cortex. This activation was not mediated by reaction time, suggesting that it was not driven by difficulty or conflict in choosing a rating.

Paracingulate cortex has previously been implicated in decision making for simple choices, where its activity is related to subsequent decisions or comparisons between options (Hampton et al., 2008; Wunderlich et al., 2009; Hare et al., 2011); it is also implicated in controlling social behavior in nonhuman primates (Hadland et al., 2003; Rudebeck et al., 2006). Paracingulate activation in the present study may therefore reflect the formation of an initial rapid decision about the merits of each particular partner in terms of his or her potential suitability for future romantic interactions.

The signal in the paracingulate cortex is unlikely to reflect a memory-based comparison in which the relative merits of a particular partner are computed with reference to other faces seen previously; because face presentation during the presession was not in the same order as dates at the events, such a sequence-dependent relative value code would be unable to make successful predictions at the presession about the outcomes of those subsequent interactions. Furthermore, the paracingulate activation seems unlikely to be related exclusively to encoding expected future reward, given that brain areas known to contribute specifically to encoding anticipated rewards, such as the VMPFC and ventral striatum (McClure et al., 2003; O'Doherty et al., 2003a; Knutson and Cooper, 2005; Montague et al., 2006), were not found to be significantly predictive of subsequent outcomes, indicating that expected future reward signals alone do not appear to drive predictions about the outcome of romantic interactions.

The fact that the VMPFC did not significantly predict decisions in this task could be accounted for in terms of a well documented role for the medial orbitofrontal cortex and VMPFC more generally in the hedonic evaluation of outcomes (experienced utility), and in the encoding of the experienced value of face stimuli in particular (O'Doherty et al., 2003b; O'Doherty, 2007; Cloutier et al., 2008). Activity in the VMPFC in the present study was found to be strongly correlated with attractiveness ratings for the faces, and subsequent inspection of the response patterns indicated that activity in this area was largely driven by partners of very high physical attractiveness. Thus, activity in this region can be interpreted as reflecting evaluation of the experienced utility of the face presented, as opposed to reflecting a computation about the merits of that partner for a potential future romantic interaction. Consistent with this hypothesis, the region of VMPFC found in the present study to be driven by

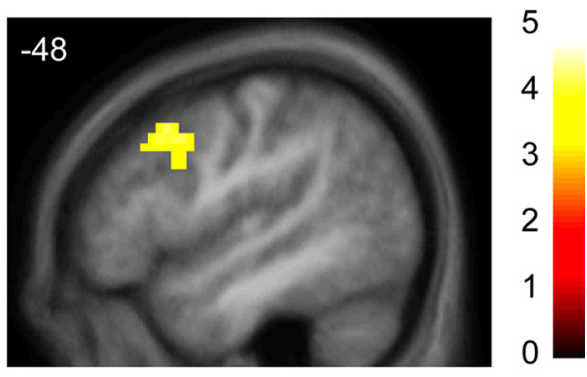

5
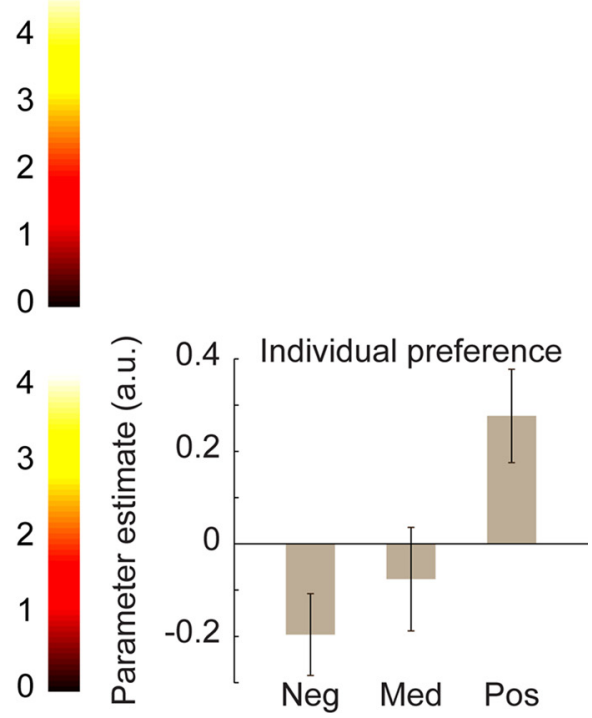

Figure 5. Neural correlation with Know ratings. $\boldsymbol{A}$, Brain regions positively correlated with Know ratings. $\boldsymbol{B}$, Brain regions (within-participant). Error bars indicate SEM across participants. All images thresholded at $p<0.001$ voxelwise with extent threshold set to control whole-brain FWE at $p<0.05$. Color bars indicate $t$ statistic. Coordinates in ICBM/MNI space.

attractiveness but not to have predictive power for subsequent decisions overlaps with that found in a recent study to be involved in the experienced value of attractive faces, as distinct from a region of VMPFC involved in computing decision values (Smith et al., 2010).

The nonlinearity in the VMPFC response is also consistent with some earlier studies on how this region responds specifically to facial attractiveness; these studies have found elevated response for the VMPFC to unattractive and attractive faces relative to middle-attractiveness faces in men only (Winston et al., 2007; Liang et al., 2010). These studies have interpreted such a pattern in terms of the relevance or salience of attractiveness to individual participants, and one interesting direction for future research might be to examine how VMPFC and paracingulate response patterns might vary with individuals' relative weight on facial attractiveness in their evaluations.

Another component of speed-dating decisions operated at the level of the interaction between specific participants and potential partners. This component was related more to likability than attractiveness; participants were more likely than others to decide to pursue a partner if they found her more likable than others did. Participants were also more likely to pursue partners for whom they had greater RMPFC activation, and this region was correlated not with partners who were desired by everybody, but with those who were especially desirable to specific participants. 
This region of RMPFC has not been specifically linked to evaluations of desirability, and so these data suggest a novel role for RMPFC in social decisions. One possible explanation of this region's activation is in its idiosyncratic response to a single partner across participants, suggesting a judgment relative to each participant. Other studies have linked this region specifically to thinking about others' mental states and to comparisons between the self and others, especially others perceived to be similar (Amodio and Frith, 2006; Mitchell et al., 2006; Van Overwalle, 2009). The current findings could thus reflect a role for RMPFC in evaluating the degree of similarity between a participant and a potential partner, which in turn could elicit greater individual interpersonal attraction in real-world speed dating (Byrne, 1971; Montoya et al., 2008).

It is important to note that the initial findings reported here implicating different regions of DMPFC in predicting subsequent outcomes of romantic interactions will need to be followed up with much more systematic computational-model-based studies aimed at uncovering the underlying computational processes during the initial evaluations and FI ratings. An interesting future direction would be to experimentally vary partner attractiveness over time to see how the brain might encode some judgment of overall potential value and potentially distinguish between different computational models of evaluation (cf. Behrens et al., 2008). Participants' decisions were also likely influenced by practical limitations in our design requiring participants to choose at least $50 \%$ of their potential partners, as well as the potential discomfort of explicitly rating partners for an experimenter; further work should evaluate how these constraints affect the relationship between rapid evaluations and the decisions themselves.

Together, these results suggest novel roles for these regions of DMPFC in real-world social decision making. Instead of being guided by a single all-encompassing judgment of desirability, real-world romantic decisions are strongly guided by multiple distinct, rapid evaluations, and their outcome can be significantly predicted on the basis of those evaluations. Activations in the paracingulate cortex and RMPFC, and not the VMPFC, are the most predictive of later real-world interactions, relating, respectively, to consensus and idiosyncratic components of these evaluations.

Judgments about romantic relationships thus seem to be formed within seconds of seeing a potential partner, but also depend on a complex mix of evaluations about physical and psychological compatibility implemented within DMPFC. Our findings build on an accumulating literature implicating the DMPFC as a key element in the machinery of human social cognition, and further demonstrate that rapid computations within this area have real-world consequences for the outcome of social interactions.

\section{References}

Adolphs R, Tranel D, Damasio AR (1998) The human amygdala in social judgment. Nature 393:470-474.

Aharon I, Etcoff N, Ariely D, Chabris CF, O'Connor E, Breiter HC (2001) Beautiful faces have variable reward value: FMRI and behavioral evidence. Neuron 32:537-551.

Ambady N, Rosenthal R (1992) Thin slices of expressive behavior as predictors of interpersonal consequences: a meta-analysis. Psych Bull 111:256274.

Amodio DM, Frith CD (2006) Meeting of minds: the medial frontal cortex and social cognition. Nat Rev Neurosci 7:268-277.

Behrens TE, Hunt LT, Woolrich MW, Rushworth MF (2008) Associative learning of social value. Nature 456:245-249.

Bray S, O’Doherty J (2007) Neural coding of reward-prediction error sig- nals during classical conditioning with attractive faces. J Neurophysiol 97:3036-3045.

Byrne D (1971) The attraction paradigm. New York: Academic.

Cloutier J, Heatherton TF, Whalen PJ, Kelley WM (2008) Are attractive people rewarding? Sex differences in the neural substrates of facial attractiveness. J Cogn Neurosci 20:941-951.

Deichmann R, Gottfried JA, Hutton C, Turner R (2003) Optimized EPI for fMRI studies of the orbitofrontal cortex. Neuroimage 19:430-441.

Eastwick PW, Finkel EJ (2008) Sex differences in mate preferences revisited: do people know what they initially desire in a romantic partner? J Pers Soc Psychol 94:245-264.

Eastwick PW, Finkel EJ, Mochon D, Ariely D (2007) Selective versus unselective romantic desire: not all reciprocity is created equal. Psychol Sci $18: 317-319$.

Finkel EJ, Eastwick PW (2008) Speed-dating. Curr Dir Psychol Sci 17:193-197.

Finkel EJ, Eastwick PW (2009) Arbitrary social norms influence sex differences in romantic selectivity. Psychol Sci 20:1290-1295.

Finkel EJ, Eastwick PW, Matthews J (2007) Speed-dating as an invaluable tool for studying romantic attraction: a methodological primer. J Soc Pers Relat 14:149-166.

Gläscher J (2009) Visualization of group inference data in functional neuroimaging. Neuroinformatics 7:73-82.

Hadland KA, Rushworth MF, Gaffan D, Passingham RE (2003) The effect of cingulate lesions on social behaviour and emotion. Neuropsychologia 41:919-931.

Hamermesh D, Biddle J (1994) Beauty and the labor market. Am Econ Rev 84:1174-1194.

Hampton AN, Bossaerts P, O'Doherty JP (2008) Neural correlates of mentalizing-related computations during strategic interactions in humans. Proc Natl Acad Sci U S A 105:6741-6746.

Hare TA, Schultz W, Camerer CF, O’Doherty JP, Rangel A (2011) Transformation of stimulus value signals into motor commands during simple choice. Proc Natl Acad Sci U S A 108:18120-18125.

Hassin R, Trope Y (2000) Facing faces: studies on the cognitive aspects of physiognomy. J Pers Soc Psychol 78:837-852.

Kenny DA (1994) Interpersonal perception: a social relations analysis. New York: Guilford.

Kenny DA, Albright L (1987) Accuracy in interpersonal perception: a social relations analysis. Psych Bull 102:390-402.

Kenny DA, West TV, Malloy TE, Albright L (2006) Componential analysis of interpersonal perception data. Pers Soc Psychol Rev 10:282-294.

Knutson B, Cooper JC (2005) Functional magnetic resonance imaging of reward prediction. Curr Opin Neurol 18:411-417.

Kriegeskorte N, Simmons WK, Bellgowan PS, Baker CI (2009) Circular analysis in systems neuroscience: the dangers of double dipping. Nat Neurosci 12:535-540.

Kurzban R, Weeden J (2005) HurryDate: mate preferences in action. Evol Hum Behav 26:227-244.

Liang X, Zebrowitz LA, Zhang Y (2010) Neural activation in the "reward circuit" shows a nonlinear response to facial attractiveness. Soc Neurosci 5:320-334.

McClure SM, Berns GS, Montague PR (2003) Temporal prediction errors in a passive learning task activate human striatum. Neuron 38:339-346.

Mitchell JP, Macrae CN, Banaji MR (2006) Dissociable medial prefrontal contributions to judgments of similar and dissimilar others. Neuron 50: 655-663.

Montague PR, King-Casas B, Cohen JD (2006) Imaging valuation models in human choice. Annu Rev Neurosci 29:417-448.

Montoya RM, Horton RS, Kirchner J (2008) Is actual similarity necessary for attraction? A meta-analysis of actual and perceived similarity. J Soc Pers Relat 25:889-922.

O'Doherty JP (2007) Lights, camembert, action! The role of human orbitofrontal cortex in encoding stimuli, rewards, and choices. Ann NY Acad Sci 1121:254-272.

O’Doherty JP, Dayan P, Friston K, Critchley H, Dolan RJ (2003a) Temporal difference models and reward-related learning in the human brain. Neuron 38:329-337.

O'Doherty J, Winston J, Critchley H, Perrett D, Burt DM, Dolan RJ (2003b) Beauty in a smile: the role of medial orbitofrontal cortex in facial attractiveness. Neuropsychologia 41:147-155.

Rudebeck PH, Buckley MJ, Walton ME, Rushworth MF (2006) A role for 
the macaque anterior cingulate gyrus in social valuation. Science 313:1310-1312.

Rule NO, Moran JM, Freeman JB, Whitfield-Gabrieli S, Gabrieli JD, Ambady N (2011) Face value: amygdala response reflects the validity of first impressions. Neuroimage 54:734-741.

Satpute AB, Lieberman MD (2006) Integrating automatic and controlled processes into neurocognitive models of social cognition. Brain Res 1079:86-97.

Schiller D, Freeman JB, Mitchell JP, Uleman JS, Phelps EA (2009) A neural mechanism of first impressions. Nat Neurosci 12:508-514.

Smith DV, Hayden BY, Truong TK, Song AW, Platt ML, Huettel SA (2010) Distinct value signals in anterior and posterior ventromedial prefrontal cortex. J Neurosci 30:2490-2495.

Spezio ML, Rangel A, Alvarez RM, O’Doherty JP, Mattes K, Todorov A, Kim H, Adolphs R (2008) A neural basis for the effect of candidate appearances on election outcomes. Soc Cogn Affect Neurosci 3:344-352.

Todorov A, Mandisodza AN, Goren A, Hall CC (2005) Inferences of competence from faces predict election outcomes. Science 308:1623-1626.

Van Overwalle F (2009) Social cognition and the brain: a meta-analysis. Hum Brain Mapp 30:829-858.
Willis J, Todorov A (2006) First impressions: making up your mind after a 100-ms exposure to a face. Psychol Sci 17:592-598.

Winston JS, Strange BA, O’Doherty J, Dolan RJ (2002) Automatic and intentional brain responses during evaluation of trustworthiness of faces. Nat Neurosci 5:277-283.

Winston JS, O’Doherty J, Kilner JM, Perrett DI, Dolan RJ (2007) Brain systems for assessing facial attractiveness. Neuropsychologia 45: 195-206.

Wood D, Brumbaugh CC (2009) Using revealed mate preferences to evaluate market force and differential preference explanations for mate selection. J Pers Soc Psychol 96:1226-1244.

Worsley KJ, Marrett S, Neelin P, Vandal AC, Friston KJ, Evans AC (1996) A unified statistical approach for determining significant voxels in images of cerebral activation. Hum Brain Mapp 4:58-73.

Wunderlich K, Rangel A, O’Doherty JP (2009) Neural computations underlying action-based decision making in the human brain. Proc Natl Acad Sci U S A 106:17199-17204.

Zebrowitz LA, McDonald SM (1991) The impact of litigants' babyfacedness and attractiveness on adjudications in small claims courts. Law Hum Behav 15:603-623. 